\title{
Pharmacology
}

Chemotherapy

\section{L-Carnitine Ameliorates}

\section{L-Asparaginase-Induced Acute Liver Toxicity in Steatotic Rat Livers}

\author{
Anne Roesmann ${ }^{a}$ Mamdouh Afify ${ }^{a, d}$ Jens Panse ${ }^{b}$ Albrecht Eisert ${ }^{c}$ \\ Julia Steitz ${ }^{\text {a }}$ Rene H. Tolba ${ }^{a}$ \\ ${ }^{a}$ Institute for Laboratory Animal Science, ${ }^{b}$ Department of Oncology, Hematology and Stem Cell Transplantation, \\ and ${ }^{\mathrm{C} C e n t r a l}$ Pharmacy, University Hospital, RWTH Aachen University, Aachen, Germany; ${ }^{\mathrm{d}}$ Department of Pathology, \\ Faculty of Veterinary Medicine, Cairo University, Cairo, Egypt
}

\section{Key Words}

L-Asparaginase $\cdot$ L-Carnitine $\cdot$ Chemotherapy $\cdot$ Leukemia . Liver toxicity - Lymphoma $\cdot$ Rat model of liver reperfusion . Steatotic liver

\section{Abstract}

Background: Chemotherapy with L-asparaginase is associated with hepatotoxicity resulting in organ dysfunction in patients with preexisting liver disorders. This study investigated the protective effect of $L$-carnitine during chemotherapy in a steatotic rat liver model. Methods: Livers from nonsteatotic and steatotic rats were tested in an isolated liver reperfusion model adding $\mathrm{L}$-asparaginase and $\mathrm{L}$-carnitine to the reperfusate. Portal venous pressure (PVP), hepatic oxygen consumption, aspartate aminotransferase, lactate dehydrogenase, glutamate dehydrogenase and a-glutathione Stransferase levels were assessed. Further histopathological analysis was performed and cytotoxicity was verified in vitro. Results: L-Asparaginase induced toxicity in fatty livers whereas low toxicity was observed in normal livers. L-Carnitine induced a decline in PVP and oxygen consumption, and reduced parenchymal and mitochondrial damage in fatty livers. Cytotoxicity of L-asparaginase was not impaired by the presence of L-carnitine. Conclusions: Our study emphasizes the potential of L-carnitine to reduce L-asparaginase-induced hepatotoxicity in patients with preexisting liver disorders.

C 2013 S. Karger AG, Base

\section{Introduction}

The bacterial enzyme L-asparaginase is one of the essential components of effective chemotherapeutic combination protocols used for the treatment of acute lymphoblastic leukemia (ALL) in pediatric and adult patients $[1$, 2]. Three types of asparaginase have been used clinically: a native L-asparaginase form derived from Escherichia coli, E. coli asparaginase in a pegylated form (polyethylene glycol asparaginase), and L-asparaginase isolated from the plant bacteria Erwinia chrysanthemi [2]. The incidence of ALL is highest in children with a median age of 14 years at diagnosis but also occurs in adults beyond the age of 55 (http://seer.cancer.gov/statfacts/html/alyl.html)

\section{J. Steitz and R.H. Tolba contributed equally to this work.}

KARGER

(C) 2013 S. Karger AG, Basel

0009-3157/13/0593-0167\$38.00/0
Prof. Rene H. Tolba, MD

Director Institute for Laboratory Animal Science University Hospital, RWTH Aachen University Pauwels Strasse 30, DE-52074 Aachen (Germany) E-Mail rtolba@ukaachen.de 
with increasing incidence [3]. Thus, for instance in the USA, it is estimated that there are more than 6,000 men and women with a diagnosis of ALL in 2012.

With increasing age, the risk of additional health problems and comorbidities is also increasing. People are becoming more frequently overweight and have already preexisting liver, pancreas or kidney disorders. Although L-asparaginase is effectively used in combination chemotherapy in all children's and most of the adult's ALL protocols, the drug has severe side effects, limiting its clinical use especially in elderly patients with preexisting disorders such as hepatopathies. Beside the well-known hypersensitivity reactions, coagulopathy, gastrointestinal and renal disorders, pancreatitis and hepatotoxicity are reported as common side effects [4].

L-Asparaginase catalyzes the hydrolysis of L-asparagine to L-aspartic acid and ammonia, resulting in a deficiency of L-asparagine and consequent inhibition of malignant lymphatic cell proliferation, as lymphatic cells are not able to synthesize L-asparagine de novo [5]. Therapy with L-asparaginase is associated with impaired protein synthesis, which can cause several histological and biochemical alterations in the liver [6-8].

In the industrial world, obesity is a huge health problem; up to $75 \%$ of the obese population develop nonalcoholic steatohepatitis, and in the general population its prevalence is between 10 and 24\% [9]. Nonalcoholic steatohepatitis is commonly associated with obesity, diabetes mellitus type II, hyperlipidemia as well as viral hepatitis. Steatotic-transformed livers are more susceptible to the side effects of chemotherapy than nonsteatotic livers [10], and patients with preexisting liver disorders, such as steatosis or hepatitis $[11,12]$, experience more often complications and even fatal liver failure after chemotherapy [13]. L-Asparaginase treatment is associated with increased hepatocyte damage, which is reflected by higher serum values of liver enzymes like alanine aminotransferase (ALT) $[14,15]$ and aspartate aminotransferase (AST) [15], and an increase in lipid components [16-18] and steatosis $[19,20]$. Adults experience more hepatotoxic reactions to L-asparaginase therapy than pediatric patients receiving comparable doses [4]. Therefore, in adult patients, liver toxicity during or after L-asparaginase treatment is a frequently encountered problem [21].

L-Carnitine is a natural vitamin-like compound synthesized from lysine and is derived from both dietary sources and endogenous biosynthesis mainly in the kidney and liver [22]. It acts as a carrier of fatty acids into the mitochondria and is essential for the $\beta$-oxidation of fatty acids [23]. In previous studies, it has been shown that L- carnitine was able to reduce liver damage in patients with nonalcoholic fatty liver disease [24-26]. In addition, it has been shown that cardiac and renal toxicity induced by chemotherapeutics like cisplatin and doxorubicin could be reduced by the addition of L-carnitine [27, 28]. Furthermore, L-carnitine was shown to have some properties to scavenge oxygen free radicals and to protect mitochondria in a rat model of hepatocarcinogenesis [25].

Given the possible organ-protective role of L-carnitine in chemotherapy-induced organ toxicity, we investigated here whether L-carnitine could also reduce L-asparaginase-induced hepatotoxicity in a previously established isolated steatotic rat liver reperfusion model [29].

\section{Materials and Methods}

\section{Animals}

All experiments were performed in accordance with the Federal German Law regarding the protection of animals. The Principles of Laboratory Animal Care (NIH publication No. 85-23, revised 1985) were followed. Male Wistar rats (Charles River GmbH, Sulzfeld, Germany) weighing between 225 and 300 g were studied. The animals were housed according to the guidelines of the Federation of European Laboratory Animal Science Associations. Water and standard rat diet (Sniff, Soest, Germany) was given ad libitum.

\section{Experimental Induction of Steatosis}

Liver steatosis was induced using a modified protocol [30]. The rats in the steatosis groups were fasted for 2 days, while tap water was available ad libitum. Thereafter, animals received a special diet for the next 3 days (C1000; Altromin, Lage, Germany). The diet was enriched which carbohydrates and fat free, and induced mildto-moderate (30-40\%) steatosis in the liver.

\section{Explanted Liver Reperfusion Model and Treatment}

Male Wistar rats were anesthetized by inhalation anesthesia using isoflurane (mean concentration 1.5 vol\%; Abbott $\mathrm{GmbH}$ and Co. KG, Wiesbaden, Germany). The abdomen was opened by midline incision with bilateral subcostal extensions, and all ligamentous attachments were surgically removed. The common bile duct was isolated and then cannulated with a 24-gauge polyethylene tube (Braun Melsungen AG, Melsungen, Germany) to collect total bile flow during isolated reperfusion. The hepatic artery was doubly ligated and then divided. The portal vein was cannulated with a 14-gauge polyethylene tube (Braun Melsungen AG) and then rinsed via the portal vein with $60 \mathrm{ml}$ of cold $\left(4^{\circ} \mathrm{C}\right) 0.9 \%$ saline solution (DeltaSelect GmbH, Dreieich, Germany). The suprahepatic vena cava was cut above the diaphragm directly after the start of perfusion, in order to avoid a high venous pressure due to outflow obstruction. Explanted livers were retrieved and then stored ex vivo in $125 \mathrm{ml}$ of ice-cold saline solution for $45 \mathrm{~min}$ at $4^{\circ} \mathrm{C}$. The phrenic veins were all ligated and the suprahepatic vena cava was cannulated with a short 14-gauge catheter.

The livers were then immediately reperfused in vitro for 120 min in a recirculating system at a constant flow of $3 \mathrm{ml}$ per gram 
of liver mass per minute with $200 \mathrm{ml}$ of oxygenated $\left(95 \% \mathrm{O}_{2}, 5 \%\right.$ $\mathrm{CO}_{2}$ ) Krebs-Henseleit buffer at $37^{\circ} \mathrm{C}$, as described in detail by Minor et al. [31].

For the treatment with L-asparaginase $(1,000 \mathrm{U} / \mathrm{kg}$ body weight, BW; Medac, Wedel, Germany), L-carnitine (20 mM) was added to $200 \mathrm{ml}$ of Krebs-Henseleit buffer (Sigma-Aldrich, Deisenhofen, Germany) and used for reperfusion of the isolated rat liver.

\section{Assessment of Liver Graft Damage and Function}

Portal venous pressure (PVP) was continuously determined using a water column connected to the portal vein inflow catheter throughout the reperfusion time.

Hepatic oxygen consumption was measured to assess the functional recovery of the reperfused livers. Perfusate samples were collected from both portal inflow and venous outflow, followed by measurement of oxygen content using a $\mathrm{pH}$ blood gas analyzer (Acid Base Laboratory, ABL 500; Radiometer Copenhagen, Denmark). The differences between portal and venous sites were calculated to obtain oxygen uptake of the liver/hepatocytes. The values were expressed as microliters per gram of liver per minute according to transhepatic flow and liver mass.

\section{Measurement of Hepatic Enzyme Release}

To characterize hepatic damage, the effluent was intermittently collected at 5, 15, 30, 45, 60, 75, 90, 105 and 120 min of reperfusion, and the release of liver-specific enzymes was analyzed. These particular enzymes are AST, glutamate-dehydrogenase (GLDH) and lactate-dehydrogenase (LDH).

AST and LDH were measured to assess hepatic injury using standard enzymatic methods and were quantified photometrically (Vitros 250; Ortho-Clinical-Diagnostics, Johnson and Johnson Company, New Jersey, N.J., USA). GLDH was analyzed as a parameter of severe hepatocellular injury at the mitochondrial level and measured with a commercially available photometric test kit (Analyticon Biotechnologies AG, Lichtenfels, Germany) according to the manufacturer's instructions (Tecan Infinite M200, Crailsheim, Germany).

Levels of a-glutathione S-transferase ( $a$-GST) in the perfusate were measured by an enzyme-linked immunoabsorbent assay (ELISA) using a rat $\alpha$-GST ELISA kit (Biotrend, Köln, Germany). The enzyme substrate reaction was measured spectrophotometrically using a microplate reader (Tecan infinite M200) according to the manufacturer's instructions.

\section{Histopathology}

Specimens from the left liver lobe were taken and immediately immersed in $4 \%$ neutral buffered formaldehyde solution, processed in different grades of xyline and alcohol and embedded in paraffin. Liver sections of $4-\mu \mathrm{m}$ thickness were generated and stained with hematoxylin and eosin using standard protocols.

Samples were evaluated for the presence of pathological findings. Therefore, degenerative changes as hepatocellular vacuolization, necrosis and Kupffer cell and endothelial cell swelling were investigated.

\section{Cell Culture and Cytotoxicity Assay}

Ramos human Burkitt's lymphoma cells and Jurkat human acute T-cell leukemia cells were cultured in RPMI 1640 (without phenol red) containing $10 \%$ heat-inactivated FBS, 2 mM L-glutamine, $0.1 \mathrm{~mm}$ nonessential amino acids, $100 \mathrm{IU} / \mathrm{ml}$ penicillin, 100 $\mathrm{mg} / \mathrm{ml}$ streptomycin and $1 \mathrm{~mm}$ sodium pyruvate. All cells were cultured at $37^{\circ} \mathrm{C}$ in water-saturated air supplemented with $5 \%$ $\mathrm{CO}_{2}$. Culture media were changed every 3 days. Cells were passaged twice a week. Cell numbers were calculated with the help of a Neubauer cell counting chamber. For cytotoxicity assays, exponentially growing cells were harvested and plated in 96-well tissue culture plates at an initial density of 10,000 cells/well seeded in $200 \mu \mathrm{l}$ of culture medium and incubated for $48 \mathrm{~h}$ at $37^{\circ} \mathrm{C}$ and $5 \%$ $\mathrm{CO}_{2}$.

On the 2nd day, fresh medium containing increasing concentrations of L-asparaginase with/without L-carnitine were added. As a control, culture medium only was used (100\% survival). Cells were cultured for $24,48,72$ or $96 \mathrm{~h}$. Cell growth was measured by the colorimetric XTT assay measuring mitochondrial activity and therefore the viability of the cells in the well. XTT solution was added to the cells according to the assay instructions and color change was evaluated after $2 \mathrm{~h}$ with an ELISA reader at a wavelength of $450 \mathrm{~nm}$ with a reference wavelength of $620 \mathrm{~nm}$.

Means of sample and control (without L-asparaginase $=100 \%$ survival) triplicates of optical density (OD) $450-620 \mathrm{~nm}$ and mean $5 \mathrm{U}$ L-asparaginase ( $=0 \%$ survival) were generated.

Survival was calculated for each concentration as follows: survival $(\%)=($ mean of OD sample - mean of OD $0 \%) /($ mean of OD control $100 \%$ - mean of OD $0 \%) \times 100$.

\section{Statistical Analysis}

All results are expressed as means \pm SEM or SD. Statistical analysis was performed using GraphPad Prism Software version 5.01 (GraphPad Software Inc., San Diego, Calif., USA). Comparisons between the experimental groups were performed by 2 -way analysis of variance (ANOVA) for the following parameters: PVP, AST, LDH, GLDH, a-GST and oxygen consumption followed by Bonferroni post hoc tests. The differences were considered statistically significant when $\mathrm{p}<0.05$. The area under the curve (AUC) was analyzed as a method based on cumulative measurement of perfusate concentrations. Prism calculates AUC using the trapezoid rule and compares the results with the other groups for investigation.

\section{Results}

In initial studies, we first established the optimal concentration of L-asparaginase by testing 200, 500 and 1,000 $\mathrm{U} / \mathrm{kg} \mathrm{BW}$, i.e. concentrations routinely used under clinical conditions for the treatment of leukemia and lympho$\mathrm{ma}$. The use of $1,000 \mathrm{U} / \mathrm{kg}$ BW showed hereby clearly signs of liver toxicity and was selected for subsequent studies. In addition, we also established the most favorable concentration of L-carnitine by testing various concentrations, i.e. $5,10,15$ and $20 \mathrm{~mm}$ in a dose escalation study, to estimate its effects on liver recovery and function. Due to insignificant improvements between the 10and $20-\mathrm{mm}$ treatment groups, suggesting that a plateau was reached, we decided to use a concentration of $20 \mathrm{mM}$ L-carnitine in the present study. The final study design of 


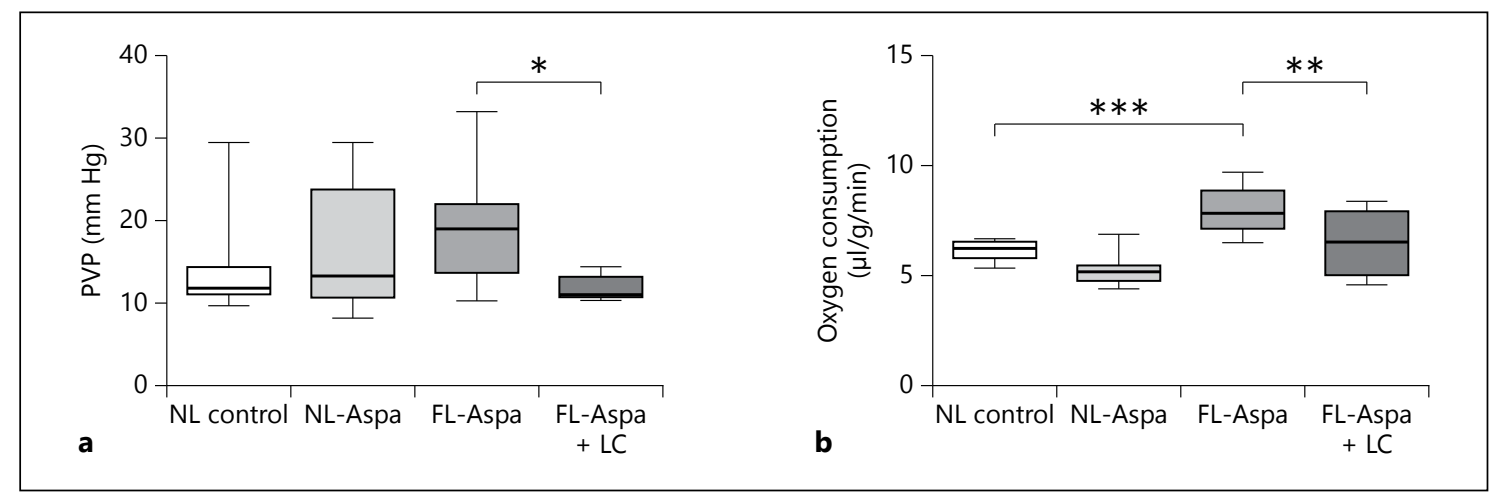

Fig. 1. Measurement of PVP (a) and oxygen consumption (b) as parameters of hepatic viability at 120 min of reperfusion time. Means $\pm \mathrm{SEM} ;{ }^{*} \mathrm{p}<0.05,{ }^{* *} \mathrm{p}<0.01,{ }^{* * *} \mathrm{p}<0.001 . \mathrm{LC}=\mathrm{L}-$ Carnitine.

Table 1. Experimental design

\begin{tabular}{lllll}
\hline Group & $\begin{array}{l}\text { Diet } \\
\text { (induction of steatosis) }\end{array}$ & $\begin{array}{l}\text { L-Asparaginase, } \\
\text { U/kg BW }\end{array}$ & $\begin{array}{l}\text { L-Carnitine, } \\
\mathrm{mM}\end{array}$ & $\begin{array}{l}\text { Animals, } \\
\mathrm{n}\end{array}$ \\
\hline NL control & normal & - & - & 7 \\
NL-Aspa & normal & 1,000 & - & 9 \\
FL control & carbohydrate enriched & - & - & 6 \\
FL-Aspa & carbohydrate enriched & 1,000 & - & 8 \\
FL-Aspa + L-carnitine & carbohydrate enriched & 1,000 & 20 & 7 \\
\hline
\end{tabular}

${ }^{1}$ For parameter levels similar to the NL control, only histopathological results are shown.

the present investigation included the normal liver (NL control), fatty liver (FL control), L-asparaginase-treated normal liver (NL-Aspa) and fatty liver (FL-Aspa) groups. As the group of interest, the FL-Aspa group received in addition L-carnitine (table 1). Since the parameters assessed in the FL control group were similar to those in the $\mathrm{NL}$ control group, only the NL control group is shown unless indicated otherwise. To determine the degree of hepatic cell viability in the different treatment groups, PVP and hepatic oxygen consumption were measured during reperfusion (fig. 1). PVP, an indicator of vascular resistance of the liver during reperfusion, was increased in the FL-Aspa group, but the addition of L-carnitine significantly reduced the PVP level to the NL group level ( $\mathrm{p}<0.05$, fig. 1a).

The measurement of hepatic oxygen consumption, an indicator of viability and the oxidative stress response of the reperfused livers, demonstrated that fatty livers showed increased oxygen consumption compared to normal livers $(\mathrm{p}<0.01)$. Again, the supplementation of
$20 \mathrm{mM}$ L-carnitine was able to decrease the values almost to the levels of normal livers ( $p<0.05$; fig. $1 b$ ).

For the characterization of liver damage, we distinguished between parenchymal- and mitochondrial-related liver damage by analyzing the release of liver-specific enzymes at different time points of reperfusion in the perfusate. Hereby, AST, LDH and $\alpha$-GST were measured to assess parenchymal hepatic injury whereas GLDH was used as a parameter of severe hepatocellular injury at the mitochondrial level (fig. 2).

Statistically significant differences in enzyme levels were detected starting at $105 \mathrm{~min}$ of reperfusion time. However, no differences were found during the early reperfusion period (until $75 \mathrm{~min}$ ). Representative data are shown after $120 \mathrm{~min}$ of reperfusion where treatment of $\mathrm{L}$-asparaginase in fatty livers (FL-Aspa) resulted in an increase in AST and LDH (fig. 2a, b). Notably, treatment with L-carnitine was able to reduce high levels of AST and $\mathrm{LDH}$ in fatty livers to the level almost equal to the $\mathrm{NL}$ control group (ALT: $\mathrm{p}<0.01$ LDH: $\mathrm{p}<0.001$ ). 


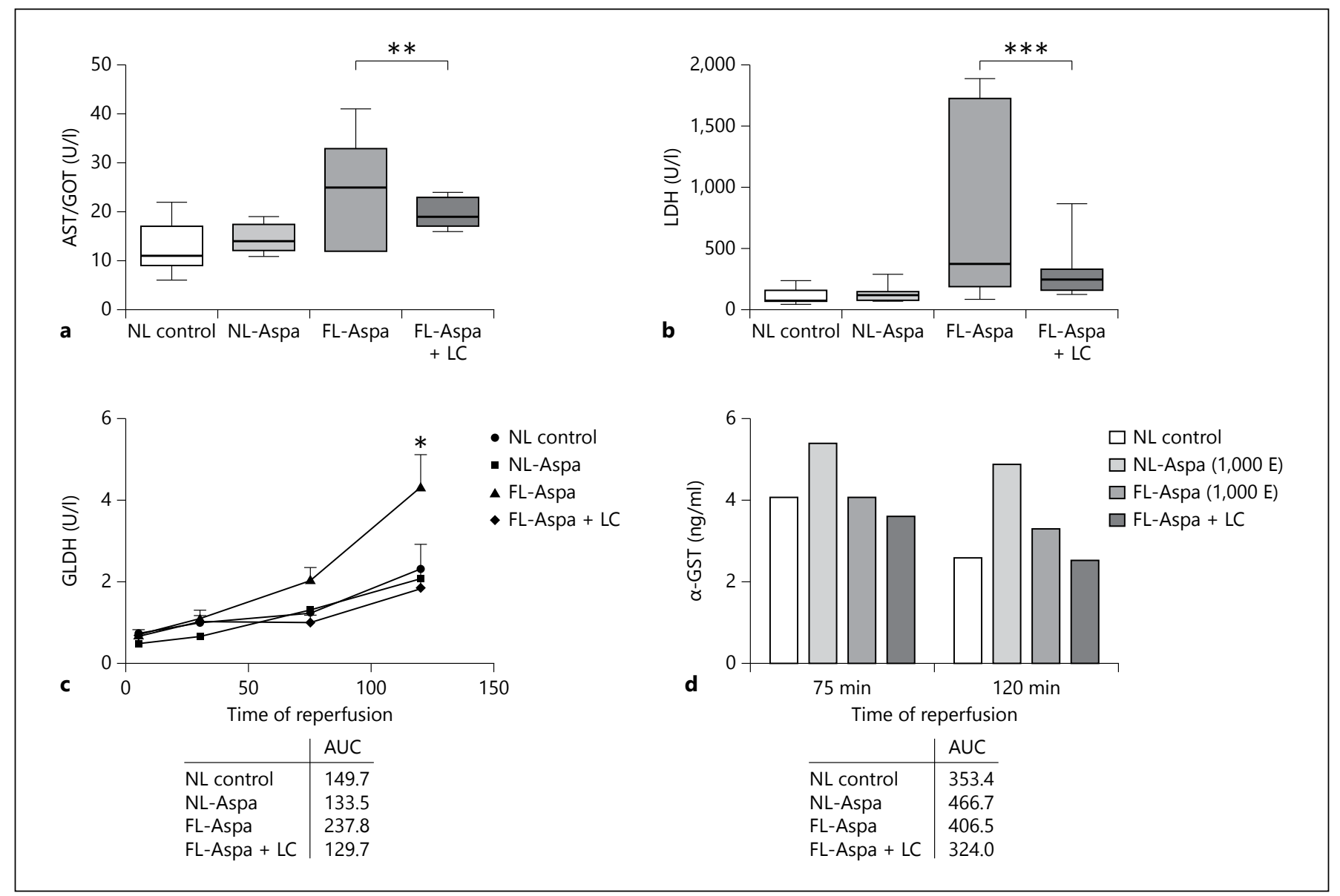

Fig. 2. Release of AST (a) and LDH (b) as signs of parenchymal liver damage at $120 \mathrm{~min}$ of reperfusion time. c GLDH levels as a parameter of mitochondrial liver damage upon reperfusion and calculations of AUC. $\mathbf{d}$ Release of $\alpha$-GST as a sensitive and liver- specific indicator for hepatocellular injury at 75 and $120 \mathrm{~min}$ of reperfusion and calculations of AUC. Means \pm SEM; for $\alpha$-GST SEM bars are not visible because values are too small; * $\mathrm{p}<0.05$, ${ }^{* *} \mathrm{p}<0.01,{ }^{* * *} \mathrm{p}<0.001 . \mathrm{LC}=\mathrm{L}-$ Carnitine.
Similar results were detected by measuring GLDH and $\alpha$-GST release. As shown in figure $2 c, d$, the positive effect of L-carnitine was noticeable at $75 \mathrm{~min}$ but more prominent at $120 \mathrm{~min}$ of reperfusion. Levels of GLDH and a-GST in the FL-Aspa + L-carnitine group were significantly lower compared with the control groups $(\mathrm{p}<$ 0.001). The AUC differences were even more prominent (tables below figure $2 c, d$ ). Because of its short half-life in plasma (90 min), $\alpha$-GST is a very sensitive and liver-specific indicator of hepatocellular injury.

Remarkably, results of all liver parameters demonstrated that the addition of L-carnitine improved the liver condition after treatment with the chemotherapeutic agent L-asparaginase in fatty livers.

Histopathological analysis was performed to visualize the degree of liver damage induced by L-asparaginase in fatty livers. Therefore, liver samples of normal and fatty livers treated with and without L-asparaginase and L-carnitine were stained with hematoxylin and eosin and analyzed for the appearance of pathological findings. These included existence of vacuoles, hepatocellular necrosis and Kupffer cell reactions. Representative data are shown in figure 3. Liver samples from normal liver with or without L-asparaginase treatment were comparable. Liver structure was intact while both showed approximately $20 \%$ of vacuoles in the tissue. As a result of the carbohydrate-enriched diet, livers in the FL-control and FL-Aspa groups showed a high amount of vacuoles and even ballooning of hepatocytes while L-asparaginase treatment enhanced the amount of vacuoles and bloated or even ruptured hepatocytes. Such changes involve $50-80 \%$ of hepatocytes. Kupffer cell reactions and endothelial cell 


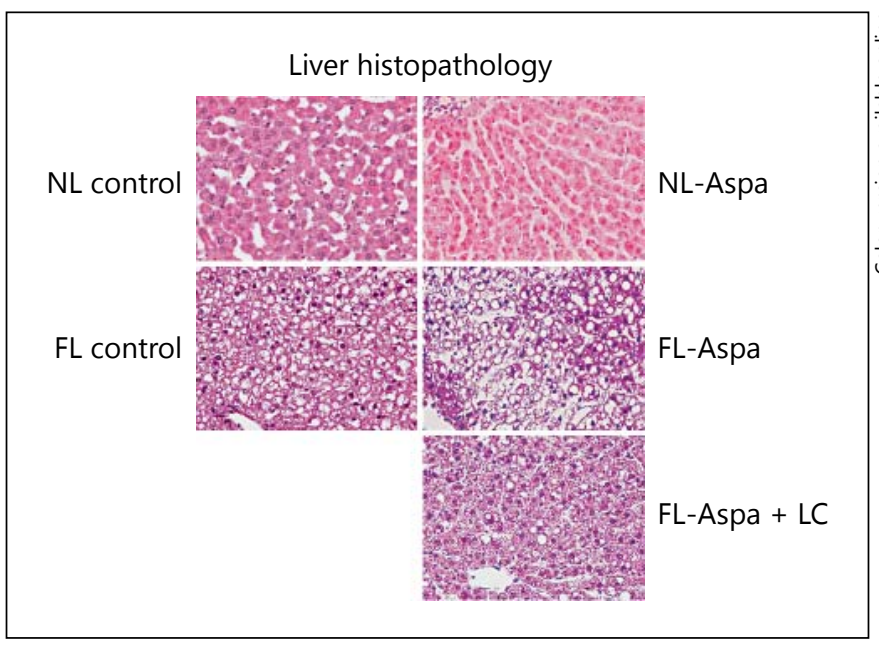

Fig. 3. HE staining of liver sections. $\times 200 . \mathrm{LC}=\mathrm{L}$-Carnitine.

swelling were also noted. Interestingly, the addition of Lcarnitine was able to reduce these pathological findings up to $20-40 \%$.

To assure that the efficiency of L-asparaginase as a lymphotoxic chemotherapeutic agent is not impaired by L-carnitine, in vitro cytotoxicity assays were performed. Therefore, various concentrations of L-asparaginase were added to Ramos human Burkitt's lymphoma cells and Jurkat human acute T-cell leukemia cells and the cytotoxic effect was determined after 24, 48, 72 and $96 \mathrm{~h}$.

In parallel, cells treated with $\mathrm{L}$-asparaginase received also L-carnitine at a final concentration of $100 \mu \mathrm{M}$. Representative results are shown in figure $4 a$, b. Testing of various concentrations of L-asparaginase showed no significant differences in cytotoxicity when L-carnitine was added. In a second set of experiments, excess L-carnitine up to a concentration of $20 \mathrm{mM}$ together with constant Lasparaginase levels $(0.315 \mathrm{U} /$ well $)$ did not change the cytotoxic effect of L-asparaginase (fig. $4 \mathrm{c}, \mathrm{d}$ ).

\section{Discussion}

The demographic transition in the human population with increasing numbers of people older than 65 years results in a higher prevalence of cancer, including lymphatic leukemia and lymphoma [3]. Health problems also increase with increasing age. Fatty livers or hepatic steatosis are common in populations around the world and often a result of obesity, alcohol consumption, diabetes or medical treatment $[32,33]$. In this group of patients, chemotherapy is problematic, since chemotherapeutic agents are often toxic to the liver and other organs. At the time of discovery and identification of L-asparaginase in the early 1960s, there were no reports about the side effects of L-asparaginase [34]. Until now, various side effects are known, such as anaphylactic reactions and - as a result of impaired protein synthesis - hepatic dysfunction in particular [35-37]. Hepatic dysfunction occurs more frequently in patients with steatotic liver disease treated with L-asparaginase [13]. In an experimental L-carnitine deficiency rat model, Al-Majed [38] could demonstrate that the injection of the inactive isomer D-carnitine induced hepatotoxicity after cisplatin treatment. Here, the supplementation of $\mathrm{L}$-carnitine was able to reduce these toxic effects.

Therefore, the aim of the present study was to investigate the ability of L-carnitine to ameliorate L-asparaginase-induced acute liver toxicity in a steatotic liver rat model.

Several studies investigating fatty livers as organ donors in liver transplantation have shown impaired vascular conductivity, most likely caused by impaired sinusoidal endothelial cells [39]. This leads to impaired microcirculation and deteriorating tissue integrity as a result of insufficient oxygenation [40-42]. These circulation-related effects are amplified by oxygen free radicals. Compromised vascular perfusion characteristics after L-asparaginase treatment of fatty livers were corroborated in the present study by an abnormally increased PVP under constant flow characteristics. Portal reflow, however, was significantly improved by the addition of L-carnitine to the reperfusion medium. These results are in line with previous results of our group, where the addition of L-carnitine to the organ preservation solution of liver grafts was also able to reduce the PVP during reperfusion [29]. A possible mechanism to explain the rapid-acting effect of L-carnitine to reduce vascular resistance is through the direct anti-oxidative effect and the regulation of the peroxisome proliferator-activated receptor alpha (PPARa), as recently shown by Li et al. [43].

As a viability parameter, we analyzed oxygen consumption of the liver during isolated liver perfusion. The FL-Aspa group showed significantly increased oxygen consumption in comparison to the NL control and NL-Aspa group. Here we could demonstrate, that the addition of $20 \mathrm{mM} \mathrm{L}$-carnitine could ameliorate the stress metabolism in steatotic livers via reducing oxygen consumption to levels equal to normal livers $(\mathrm{p}>0.05)$. 


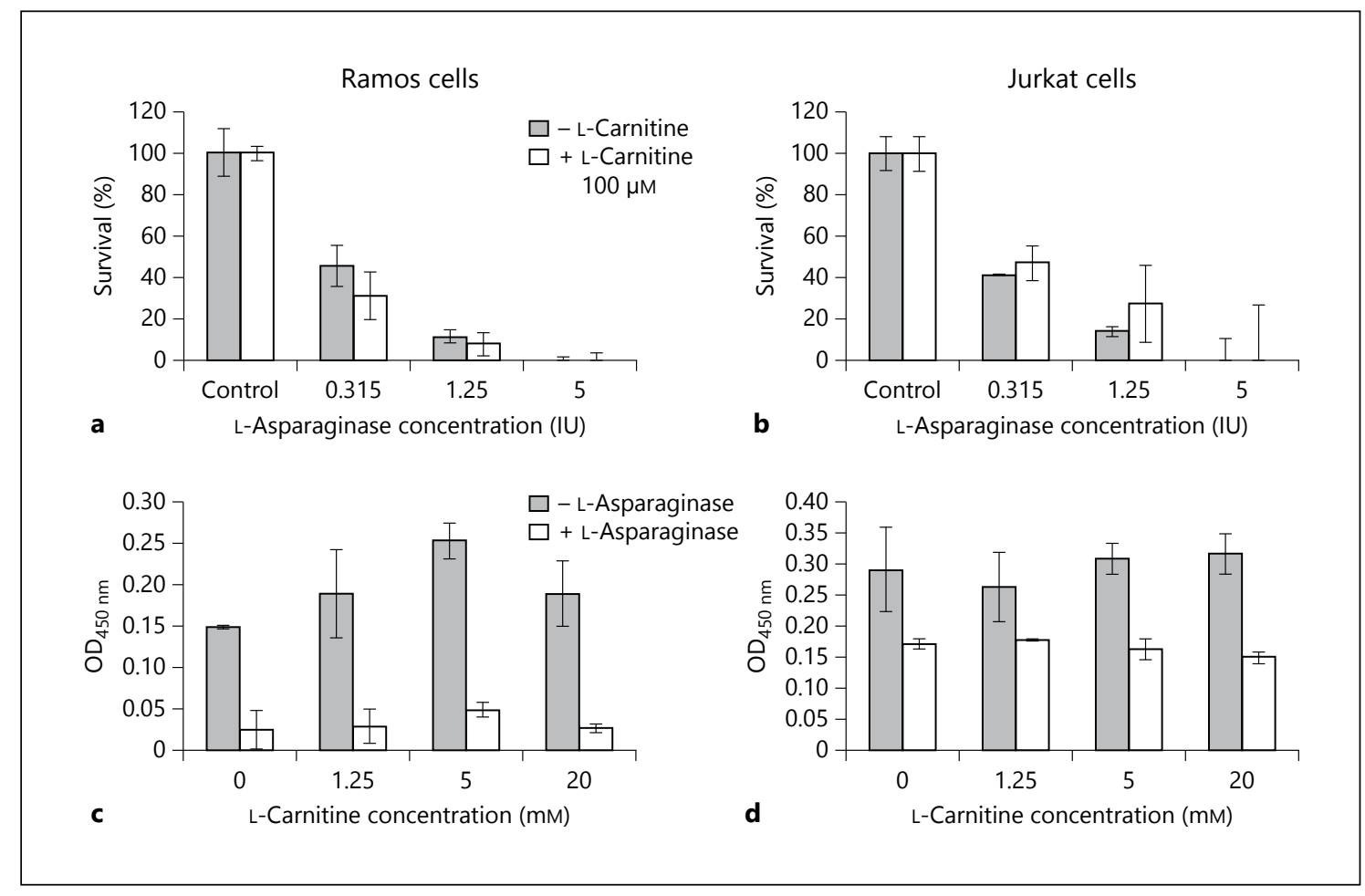

Fig. 4. Efficiency of L-asparaginase in the presence of L-carnitine. In vitro cytotoxicity is shown as percent survival of various concentrations of L-asparaginase in Ramos human Burkitt's lymphoma cells (a) and Jurkat human acute T-cell leukemia cells (b) in the pres- ence of $100 \mu \mathrm{M} \mathrm{L}$-carnitine. In vitro cytotoxicity levels are shown as $\mathrm{OD}_{450 \mathrm{~nm}}$ of L-asparaginase $(0.315 \mathrm{U} /$ well $)$ in the presence of various L-carnitine concentrations in Ramos human Burkitt's lymphoma cells (c) and Jurkat human acute T-cell leukemia cells (d).
These results were corroborated within this study by the results of the enzyme release.

The treatment of steatotic livers with L-asparaginase resulted in an increased release of $\mathrm{LDH}$ and liver-specific enzymes, such as AST and $\alpha$-GST. The release of GLDH as a marker of mitochondrial damage was significantly increased at the end of the reperfusion period. This is in correlation with the increased oxygen consumption of the FL-Aspa group. The addition of L-carnitine was able to reduce these effects to the levels of the NL control group. These results could confirm the ability of L-carnitine to reduce toxic side effects of chemotherapeutic agents, e.g. cisplatin toxicity in the kidney $[27,44]$, cisplatin-induced ototoxicity and neurotoxicity [45] and also in tilmicosin and doxorubicin toxicity in the heart $[46,47]$. In contrast, the combination of L-carnitine with mitoxantrone in a mouse solid tumor model of Ehrlich tumor did not show a beneficial effect of Lcarnitine. Here, Niang et al. [48] reported a negative effect of L-carnitine on the therapeutic outcome of mitoxantrone.

L-Carnitine Ameliorates L-AsparaginaseInduced Acute Liver Toxicity
As shown previously, L-carnitine is essential for the transport of fatty acids through the inner mitochondrial membrane and for the $\beta$-oxidation of fatty acids [49].

Histopathological analysis of the livers could clearly confirm the above observation of the beneficial effect of L-carnitine. The addition of L-carnitine to L-asparaginase-treated fatty livers could prevent toxic effects on the liver indicated by the appearance of pathological findings, which included the existence of vacuoles, Kupffer cell reactions, intralobular necrosis and intracellular edema.

These findings are in line with the current literature where we and other groups could demonstrate protective effects of L-carnitine on ultrastructural levels in various models [29, 47, 50-52].

In order to verify that the addition of L-carnitine has no negative impact on the efficiency of the chemotherapeutic drug L-asparaginase, we performed in vitro assays testing various tumor cell lines and concentrations of Lcarnitine. No negative impact of L-carnitine on the efficiency of L-asparaginase could be observed. 
In conclusion, we could demonstrate that the addition of L-carnitine could reduce L-asparaginase-induced heptotoxicity in an isolated perfused fatty rat liver model. These findings support the hypothesis that L-carnitine might have protective effects on L-asparaginase chemotherapy. This may pave the way for a novel approach to safer and less toxic chemotherapeutic treatment of patients with sensitive steatotic liver transformations.

\section{Acknowledgment}

The authors thank Martyna Wojcieszak, Pascal Paschenda and Mareike Schulz for their skilful technical assistance. This work was supported by the ForSaTum grant to R. Tolba funded by the European Union and NRW government [NRW-EU Ziel 2 Programm 2007-2013 'Regionale Wettbewerbsfähigkeit und Beschäftigung (EFRE)', Förderkennzeichen 005-0908-0112].

\section{References}

$>1$ Avramis VI, Tiwari PN: Asparaginase (native ASNase or pegylated ASNase) in the treatment of acute lymphoblastic leukemia. Int J Nanomedicine 2006;1:241-254.

$>2$ Pieters R, Hunger SP, Boos J, Rizzari C, Silverman L, Baruchel A, Goekbuget N, Schrappe M, Pui CH: L-Asparaginase treatment in acute lymphoblastic leukemia: a focus on Erwinia asparaginase. Cancer 2011;117:238-249.

$\checkmark 3$ Jemal A, Bray F, Center MM, Ferlay J, Ward E, Forman D: Global cancer statistics. CA Cancer J Clin 2011;61:69-90.

-4 Earl M: Incidence and management of asparaginase-associated adverse events in patients with acute lymphoblastic leukemia. Clin Adv Hematol Oncol 2009; 7:600-606.

$>5$ Avramis VI, Panosyan EH: Pharmacokinetic/ pharmacodynamic relationships of asparaginase formulations: the past, the present and recommendations for the future. Clin Pharmacokinet 2005;44:367-393.

6 Narta UK, Kanwar SS, Azmi W: Pharmacological and clinical evaluation of L-asparaginase in the treatment of leukemia. Crit Rev Oncol Hematol 2007;61:208-221.

$>7$ Muller HJ, Boos J: Use of L-asparaginase in childhood ALL. Crit Rev Oncol Hematol 1998;28:97-113.

$>8$ Jenkins R, Perlin E: Severe hepatotoxicity from Escherichia coli L-asparaginase. J Natl Med Assoc 1987;79:775-779.

$\checkmark 9$ Torres DM, Williams CD, Harrison SA: Features, diagnosis, and treatment of nonalcoholic fatty liver disease. Clin Gastroenterol Hepatol 2012;10:837-858.

$\checkmark 10$ Miki C, Iriyama K, Mirza DF, Mayer AD, Buckels JA, Suzuki H, McMaster P: Postperfusion energy metabolism of steatotic graft and its relation to early graft viability following liver transplantation. Dig Dis Sci 1998;43: 74-79.

$>11$ Mori T, Sugita K, Suzuki T, Ishikawa T, Kurosawa $\mathrm{H}$, Matsui A: Histopathologic features of the biopsied liver at onset of childhood Bprecursor acute lymphoblastic leukemia presenting as severe jaundice. J Pediatr Gastroenterol Nutr 1997;25:354-357.

12 Soh LT, Ang PT, Sng I, Chua EJ, Ong YW: Fulminant hepatic failure in non-Hodgkin lymphoma patients treated with chemotherapy. Eur J Cancer 1992;28A:1338-1339.
13 Bodmer M, Sulz M, Stadlmann S, Droll A, Terracciano L, Krahenbuhl S: Fatal liver failure in an adult patient with acute lymphoblastic leukemia following treatment with L-asparaginase. Digestion 2006;74: 28-32.

14 Bessho F, Kinumaki H, Yokota S, Hayashi Y, Kobayashi M, Kamoshita S: Liver function studies in children with acute lymphocytic leukemia after cessation of therapy. Med Pediatr Oncol 1994;23:111-115.

15 Cairo MS: Adverse reactions of L-asparaginase. Am J Pediatr Hematol Oncol 1982;4: 335-339.

16 Parsons SK, Skapek SX, Neufeld EJ, Kuhlman C, Young ML, Donnelly M, Brunzell JD, Otvos JD, Sallan SE, Rifai N: Asparaginase-associated lipid abnormalities in children with acute lymphoblastic leukemia. Blood 1997;89: 1886-1895.

17 Celle G, Dodero M, Pannacciulli I: The liver damaging effect of L-asparaginase - an experimental study of chronic toxicity. Eur J Cancer 1973;9:55-57.

18 Durden DL, Salazar AM, Distasio JA: Kinetic analysis of hepatotoxicity associated with antineoplastic asparaginases. Cancer Res 1983; 43:1602-1605.

19 Pratt CB, Johnson WW: Duration and severity of fatty metamorphosis of the liver following L-asparaginase therapy. Cancer 1971;28: 361-364.

20 Sahoo S, Hart J: Histopathological features of L-asparaginase-induced liver disease. Semin Liver Dis 2003;23:295-299.

21 Goekbuget N, Baumann A, Beck J, Boos J, Brueggemann M, Diedrich $\mathrm{H}$, et al: PEG-asparaginase in adult acute lymphoblastic leukemia (ALL): efficacy and feasibility analysis with increasing dose levels (abstract). Blood 2008; 112:302

22 Bremer J: Carnitine - metabolism and functions. Physiol Rev 1983;63:1420-1480.

23 Bahl JJ, Bressler R: The pharmacology of carnitine. Annu Rev Pharmacol Toxicol 1987;27: 257-277.

24 Malaguarnera M, Gargante MP, Russo C, Antic T, Vacante M, Malaguarnera M, Avitabile T, Li VG, Galvano F: L-Carnitine supplementation to diet: a new tool in treatment of nonalcoholic steatohepatitis - a randomized and controlled clinical trial. Am J Gastroenterol 2010;105:1338-1345.

25 Chang B, Nishikawa M, Nishiguchi S, Inoue M: L-Carnitine inhibits hepatocarcinogenesis via protection of mitochondria. Int J Cancer 2005;113:719-729.

26 Socha P, Horvath A, Vajro P, Dziechciarz P, Dhawan A, Szajewska H: Pharmacological interventions for nonalcoholic fatty liver disease in adults and in children: a systematic review. J Pediatr Gastroenterol Nutr 2009;48:587-596.

$>27$ Tufekci O, Gunes D, Ozogul C, Kolatan E, Altun Z, Yilmaz O, Aktas S, Erbayraktar Z, Kirkim G, Mutafoglu K, Soylu A, Serbetcioglu B, Guneri EA, Olgun N: Evaluation of the effect of acetyl L-carnitine on experimental cisplatin nephrotoxicity. Chemotherapy 2009;55: 451-459.

28 Alshabanah OA, Hafez MM, Al-Harbi MM, Hassan ZK, Al Rejaie SS, Asiri YA, SayedAhmed MM: Doxorubicin toxicity can be ameliorated during antioxidant L-carnitine supplementation. Oxid Med Cell Longev 2010;3:428-433.

29 Tolba RH, Putz U, Decker D, Dombrowski F, Lauschke H: L-Carnitine ameliorates abnormal vulnerability of steatotic rat livers to cold ischemic preservation. Transplantation 2003; 76:1681-1686.

30 Delzenne NM, Hernaux NA, Taper HS: A new model of acute liver steatosis induced in rats by fasting followed by refeeding a high carbohydrate-fat free diet. Biochemical and morphological analysis. J Hepatol 1997;26: 880-885.

31 Minor T, Akbar S, Tolba R: Value of alpha glutathione $\mathrm{S}$-transferase for in vitro evaluation of preservation injury in normal and steatotic livers. Transpl Int 2000;13(suppl 1):S551-S554.

32 Ludwig J, McGill DB, Lindor KD: Review: nonalcoholic steatohepatitis. J Gastroenterol Hepatol 1997;12:398-403.

33 Clark JM, Diehl AM: Nonalcoholic fatty liver disease: an underrecognized cause of cryptogenic cirrhosis. JAMA 2003;289:3000-3004.

$\checkmark 34$ Broome JD: Evidence that the L-asparaginase of guinea pig serum is responsible for its antilymphoma effects. I. Properties of the L-asparaginase of guinea pig serum in relation to those of the antilymphoma substance. J Exp Med 1963;118:99-120. 
35 Villa P, Corada M, Bartosek I: L-asparaginase effects on inhibition of protein synthesis and lowering of the glutamine content in cultured rat hepatocytes. Toxicol Lett 1986;32:235241.

36 Haskell CM, Canellos GP, Leventhal BG, Carbone PP, Serpick AA, Hansen HH: L-Asparaginase toxicity. Cancer Res 1969;29:974975.

37 Haskell CM: L-Asparaginase: human toxicology and single agent activity in nonleukemic neoplasms. Cancer Treat Rep 1981;65(suppl 4):57-59.

38 Al-Majed AA: Carnitine deficiency provokes cisplatin-induced hepatotoxicity in rats. Basic Clin Pharmacol Toxicol 2007;100:145150.

-39 Fukumori T, Ohkohchi N, Tsukamoto S, Satomi S: The mechanism of injury in a steatotic liver graft during cold preservation. Transplantation 1999;67:195-200.

40 Takahashi K, Hakamada K, Totsuka E, Umehara Y, Sasaki M: Warm ischemia and reperfusion injury in diet-induced canine fatty livers. Transplantation 2000;69:2028-2034.

41 Sun CK, Zhang XY, Zimmermann A, Davis G, Wheatley AM: Effect of ischemia-reperfusion injury on the microcirculation of the steatotic liver of the Zucker rat. Transplantation 2001;72:1625-1631.
42 Ijaz S, Yang W, Winslet MC, Seifalian AM: Impairment of hepatic microcirculation in fatty liver (review). Microcirculation 2003;10: 447-456.

43 Li JL, Wang QY, Luan HY, Kang ZC, Wang CB: Effects of L-carnitine against oxidative stress in human hepatocytes: involvement of peroxisome proliferator-activated receptor alpha. J Biomed Sci 2012;19:32.

44 Aleisa AM, Al-Majed AA, Al-Yahya AA, AlRejaie SS, Bakheet SA, Al-Shabanah OA, Sayed-Ahmed MM: Reversal of cisplatin-induced carnitine deficiency and energy starvation by propionyl-L-carnitine in rat kidney tissues. Clin Exp Pharmacol Physiol 2007;34: 1252-1259.

45 Gunes D, Kirkim G, Kolatan E, Guneri EA, Ozogul C, Altun Z, Serbetcioglu B, Yilmaz O, Aktas S, Mutafoglu K, Tufekci O, Erbayraktar $\mathrm{Z}$, Olgun N: Evaluation of the effect of acetyl L-carnitine on experimental cisplatin ototoxicity and neurotoxicity. Chemotherapy 2011 ; 57:186-194.

46 Kart A, Yapar K, Karapehlivan M, Citil M: The possible protective effect of L-carnitine on tilmicosin-induced cardiotoxicity in mice. J Vet Med A Physiol Pathol Clin Med 2007;54: 144-146.
47 Andrieu-Abadie N, Jaffrezou JP, Hatem S, Laurent G, Levade T, Mercadier JJ: L-Carnitine prevents doxorubicin-induced apoptosis of cardiac myocytes: role of inhibition of ceramide generation. FASEB J 1999;13:15011510.

48 Niang M, Soukup T, Zivny P, Tomsik P, Bukac J, Rezacova M, Stoklasova A, Cerman J, Sispera L: Biochemical and pharmacological effects of mitoxantrone and acetyl-L-carnitine in mice with a solid form of Ehrlich tumour. Chemotherapy 2011;57:35-42.

49 Pande SV, Parvin R: Carnitine-acylcarnitine translocase catalyzes an equilibrating unidirectional transport as well. J Biol Chem 1980; 255:2994-3001.

50 Battelli D, Bellei M, Arrigoni-Martelli E, Muscatello U, Bobyleva V: Interaction of carnitine with mitochondrial cardiolipin. Biochim Biophys Acta 1992;1117:33-36.

51 Loster H, Punzel M: Effects of L-carnitine on mechanical recovery of isolated rat hearts in relation to the perfusion with glucose and palmitate. Mol Cell Biochem 1998;185:65-75.

52 Di GC, Latteri F, Fichera C, Sorrenti V, Campisi A, Castorina C, Russo A, Pinturo R, Vanella A: Effect of acetyl-L-carnitine on lipid peroxidation and xanthine oxidase activity in rat skeletal muscle. Neurochem Res 1993; 18:1157-1162. 